\title{
Detection of lead contamination of water and VOC contamination of air using SOI micro-optical devices
}

\author{
John Saunders, Marian A. Dreher, Jack A. Barnes, \\ Cathleen M. Crudden, Jenny Du, Hans-Peter Loock* \\ Dept. of Chemistry \\ Queen's University \\ Kingston, ON, K7L 3N6, Canada \\ *hploock@chem.queensu.ca
}

\author{
Dan-Xia Xu, Adam Densmore, Rubin Ma, Siegfried \\ Janz, Martin Vachon, Jean Lapointe, André Delâge, \\ Jens Schmid, Pavel Cheben \\ Institute for Microstructural Sciences \\ National Research Council \\ Ottawa, ON, K1A 0R6, Canada
}

\begin{abstract}
Micro-photonic SOI Mach-Zehnder interferometers were coated with solid-phase micro-extraction materials derived from polydimethylsiloxane to enable sensing of volatile organic compounds of the BTEX class in air. A different coating based on functionalized mesoporous silicates is used to detect lead $\mathrm{Pb}$ (II) with a detection limit of $<100 \mathrm{ppb}$ in water.
\end{abstract}

Keywords- refractive index, chemical sensor, solid-phase microextraction, interferometer, environmental monitoring

\section{INTRODUCTION}

Silicon photonic wire interferometers and ring-resonators exhibit a high sensitivity to the ambient refractive index through the interaction of the evanescent wave of the guided mode. This sensitivity can be exploited for chemical sensing purposes, if the analyte of interest can be bound to the surface of the waveguide. For example, a specific and sensitive sensor was build by exploiting the specific binding affinity between biotin and streptavidin and using a spiral-path Mach-Zehnder interferometer as the sensing platform [1]. More recently, goat immunoglobulin $\mathrm{G}$ ( $\mathrm{IgG}$ ) was bound to the same types of interferometers and demonstrated a strong response to antigoat IgG, but not to antirabbit IgG. The response could be suppressed by blocking the sensors with bovine serum albumin [2].

In this study we expand the suite of analytes to include volatile organic compounds in air, exemplified by m-xylene, and to heavy metals in water, i.e. lead $\mathrm{Pb}$ (II). Two different microextraction films were used to enable uptake and enrichment of the analyte and to provide rudimentary chemical selectivity. By comparison to previous sensors the interactions between the analytes and the films are reversible, albeit less chemically specific and less strong. For environmental sensors reversibility of the binding process under ambient conditions is essential, since elaborate recovery of a field deployable sensor is not practical.

One microextraction film was based on polydimethyl siloxane (PDMS), which has already demonstrated a large refractive index change upon uptake of $m$-xylene from air when applied to a long-period grating sensor $[3,4]$. The second microextraction film is based on a tetrasulfide-functionalized mesoporous silicate material which has also been previously

This research was supported by the Natural Sciences and Engineering Research Council of Canada and by the National Research Council of Canada applied to a long-period grating and showed a large affinity to lead $\mathrm{Pb}(\mathrm{II})[5]$.

\section{EXPERIMENTAL}

\section{A. Microinterferometer Design}

Micro-photonic spiral-path Mach-Zehnder (MZ) interferometers made from silicon-on-insulator (SOI) waveguides are used as a sensing platform (Fig.1). Polydimethylsiloxane and tetrasulfide-functionalized mesoporous silica materials were spin-coated on the exposed arm of the interferometer to a thickness of about $4 \mu \mathrm{m}$ and 400 $\mathrm{nm}$, respectively.

The fabrication of the MZ-interferometers and their properties have been described before [2]. Briefly, the waveguides were fabricated using electron beam lithography and reactive ion etching on a SOI wafer with a $2 \mu \mathrm{m}$ layer of silica under $260 \mathrm{~nm}$ of silicon. The resulting waveguides had a height of $260 \mathrm{~nm}$ and a width of $450 \mathrm{~nm}$ and were covered either with SU-8 photoresist or with silica that was deposited by plasma enhanced chemical vapour deposition. Windows were opened to expose one of the two arms to the environment (Figure 1).

\section{B. Synthesis of the Microextraction Films}

The devices were covered with PDMS or mesoporous silicate by spin-coating. About $1.2 \mathrm{~g}$ of Sylgard 184 oligomer was prepared by mixing base and curing agent in a ratio of

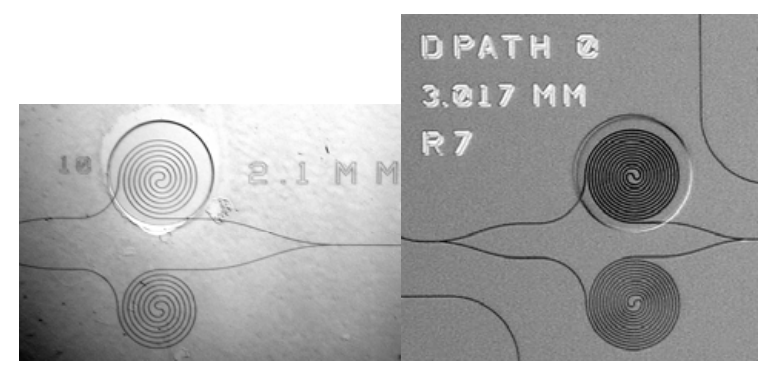

Figure 1: Microscope images showing the configuration of the balanced MachZehnder interferometer. The waveguides and lower arms of the devices was protected by SU-8 photoresist (left), and silica (right). 


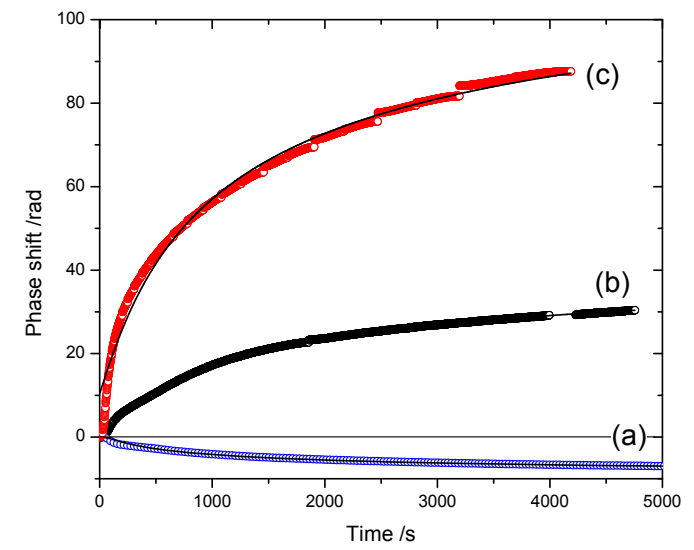

Figure 2: Uptake of m-xylene gas (11000 ppm) into uncoated chips that were protected by silica (a) or by SU8 (b). Curve (c) shows the response of a SU8 protected interferometer that was coated with $3.5 \mu \mathrm{m}$ of PDMS.

10:1. The oligomer was dissolved in $10 \mathrm{~mL}$ of hexane and cured overnight. $1 \mathrm{~mL}$ of the oligomer solution was diluted further with another $2 \mathrm{~mL}$ of hexane. The chip was spin coated with the solution at 3000 RPM for 20 seconds. The thickness of the polymer layer was determined to be approximately $3.5 \mu \mathrm{m}$ using a profilometer. The mesoporous silicate coating was synthesized as follows [5]. In a vial, $1.98 \mathrm{~g}(9.5 \mathrm{mmol})$ of tetraethoxysilane (TEOS) and $0.256 \mathrm{~g}(0.475 \mathrm{mmol})$ of bis-[3(triethoxysilyl)-propyl]-tetrasulfide (SIS) are dissolved in $3 \mathrm{ml}$ of anhydrous ethanol. After addition of $1.2 \mathrm{ml}$ of deionized water and $0.16 \mathrm{ml}$ of $6 \mathrm{M} \mathrm{HCl}$ the solution is stirred at $60^{\circ} \mathrm{C}$ for 2 hours. In a second vial, $0.719 \mathrm{~g}$ of Pluronic F127 is dissolved in $16 \mathrm{ml}$ of anhydrous ethanol and the solution is stirred for 2 hours at room temperature. The second solution is added to the first solution and the mixture is stirred at room temperature for another 2 hours. Prior to spin-coating the silica-covered SOI chips were cleaned in $\mathrm{H}_{2} \mathrm{O}_{2} / \mathrm{H}_{2} \mathrm{SO}_{4}$. The chips were coated at $3000 \mathrm{rpm}$ to a thickness of 300-500 nm. After spin-coating the chips were kept at $42 \%$ rel. humidity for 12 hours and then dried in a vacuum oven at $65^{\circ} \mathrm{C}$ for 2 hours. To extract the Pluronic surfactant, the chips were kept in refluxing acetone for 16 hours and dried in a vacuum oven for 2 hours.

\section{RESULTS}

Before the coating was applied, the interferometers were calibrated using sucrose solutions of known refractive indices. From the MZ-interferometer equation

$$
I=I_{0} \cos ^{2}\left(\pi \frac{\Delta n L}{\lambda}\right)
$$

the sensitivity of the wavelength shift, $\mathrm{d} \lambda / \mathrm{d} n_{a m b}$, and the sensitivity of the phase change, $\mathrm{d} \phi / \mathrm{d} n_{a m b}$, were found to scale linearly with the length, $L$, of its arms, as expected. By calibration with sucrose solutions we determined the sensitivity of the phase shift measurement for SU-8 coated interferometers as $4930 \mathrm{rad} / \mathrm{RIU}$ for an arm length of $2.1 \mathrm{~mm}$. This is consistent with earlier measurements using similar $2 \mathrm{~mm}$ interferometers that were covered by SU-8 (3900 rad/RIU).
The response to $\mathrm{m}$-xylene in air and water contaminants was also measured before coating the interferometer with the micro-extraction films. Both SU-8 protected devices and silica protected devices showed a strong but opposite response to xylene at its saturation pressure of $11,000 \mathrm{ppm}$ (Fig. 2). To determine the cause of this response we exposed protected and unprotected microring-resonators that were present on the same two chips to xylene at different pressures from $550 \mathrm{ppm}$ to 2200 ppm (data not shown). It was found that xylene deposited to a small extent on the unprotected microring resonators, but did not interact with the silica covered microring-resonators. On the other hand the SU-8 coated Mach-Zehnder interferometers showed a much stronger but somewhat delayed response to xylene, indicating that the SU-8 protective layer already acts as an extraction matrix, which increases its refractive index as xylene diffuses into the coating. Figure 2 shows the accumulated phase shift as a function of time. From a fit to the diffusion equation

$$
C=C_{0} \operatorname{erf}\left(\frac{d}{\sqrt{D t}}\right)
$$

with experimentally determined film thickness, $d$, (by profilometry) we estimated the diffusion coefficient as $\mathrm{D}$ (xylSU8) $=7 \times 10^{-10} \mathrm{~cm}^{2} / \mathrm{s}$.

The data shown in Fig 2 (c) corresponds to the diffusion of xylene through a layer stack of PDMS $(3.5 \mu \mathrm{m})$ on top of the SU-8 $(2 \mu \mathrm{m})$ coated device. The process is dominated by the much slower diffusion through the SU-8 layer and can be fitted to a single diffusion constant. As expected, the diffusion through PDMS is very fast and cannot be resolved on the timescale of our measurement $(D$ (xyl-PDMS $)=2 \times 10^{-6} \mathrm{~cm}^{2} / \mathrm{s}$ [6]). The uptake of xylene by SU-8 can be rationalized when considering that this epoxy compound is known to swell somewhat upon exposure to organic solvents [7] and may exhibit an affinity to aromatic compounds due to $\pi-\pi$ stacking interactions with the phenyl groups contained in SU-8.

The response of the presumably "inert" SU-8 overcoats to the analyte gas affects their performance as protective coatings for chemical sensing involving organic solvents. By contrast the SU-8 and silica overcoats have been shown to be inert in aqueous solutions in biosensing experiments, and act as reliable protective layers $[1,2]$. Since the refractive indices of the SU-8 and PDMS coatings both increase upon exposure to PDMS the sensitivity of the measurement and the attainable detection limit are compromised. The uptake of xylene into both types of coatings, however, and is still apparent at xylene concentrations below 1000 ppm (Fig. 3).

Most importantly for an environmental gas sensor, the xylene uptake process into SU-8, silica and PDMS is fully reversible at ambient temperatures and pressures. Figure 3 shows the uptake and release of xylene gas into PDMS that was cast on top of an SU-8 protected interferometer. The curve shows the transmitted intensity at a fixed wavelength of 1529.4 $\mathrm{nm}$. A detection limit of $550 \mathrm{ppm}$ was realized and a lower limit of detection of $<100 \mathrm{ppm}$ can be extrapolated considering the low noise level of the measurement. From the linear dependence of the phase shift with PDMS refractive index and xylene concentration $\left(\mathrm{d} \phi / \mathrm{d} C_{x y l}=4.3 \times 10^{-3} \mathrm{rad} / \mathrm{ppm}\right)$ and from 

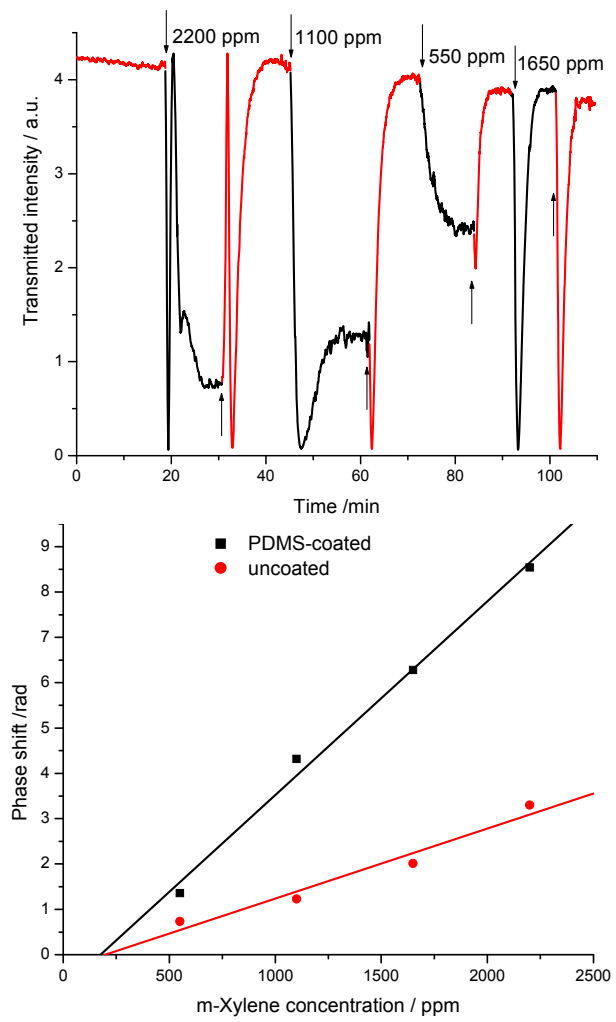

Figure 3: Uptake and release of m-xylene at different partial pressures in nitrogen into the PDMS matrix deposited onto a SU8-protected MZinterferometer. The top panel shows nearly complete reversibility when and pure nitrogen is injected. The bottom panel indicates a fairly linear response over the measured range.

the sensitivity of the $2.1 \mathrm{~mm}$ interferometer $\left(\mathrm{d} \phi / \mathrm{d} n_{a m b}=4930\right.$ $\mathrm{rad} / \mathrm{RIU})$ we calculated the change of the PDMS refractive index upon xylene uptake as $8.7 \times 10^{-7} \mathrm{RIU} / \mathrm{ppm}$. As shown before, this sensitivity can be increased by insertion of phenyl groups into the PDMS polymer [3, 4] and by using silica as a protective coating for the micro-photonic devices.

In a preliminary experiment $2.04 \mathrm{~mm} \mathrm{MZ}$-interferometers protected by a $\mathrm{SiO}_{2}$ layer were used to detect lead, $\mathrm{Pb}$ (II) in water using the $400 \mathrm{~nm}$ mesoporous silica coating as the extraction matrix. Water was directed towards the coated MZinterferometers using a capillary that expanded into a small container (volume $\sim 80 \mu \mathrm{L}$ ), which was sealed against the SOI device. The previously measured change of the coating's refractive index upon lead uptake was $\mathrm{d} n / \mathrm{d} C_{P b}=1.0-5.0 \times 10^{-6}$ RIU/ppb depending on the synthesis [5]. Given the measured sensitivity of the MZ-interferometer of $\mathrm{d} \phi / \mathrm{d} n_{a m b}=2536$ $\mathrm{rad} / \mathrm{RIU}$ we estimate that $100 \mathrm{ppb}(640 \mathrm{nM})$ solutions of lead will lead to a phase shift of 0.3-1.3 rad and should therefore be detectable. Indeed, Figure 4 shows that a solution of $100 \mathrm{ppb}$ lead not only leads to a measurable phase change but that lead uptake is also largely reversible. More measurements will be performed at higher and lower concentrations to determine the detection limit and dynamic range of the measurement.

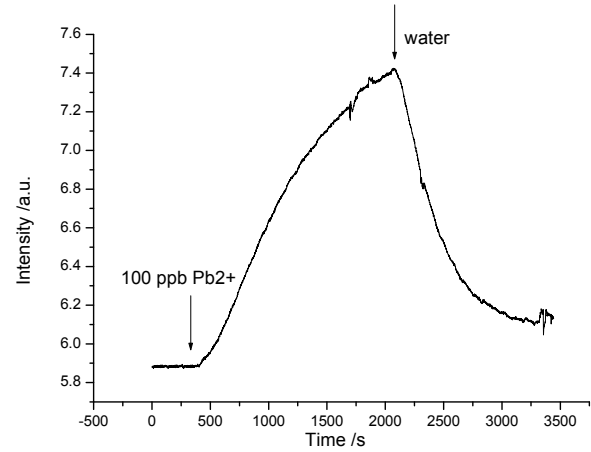

Figure 4: Uptake and release of 100 ppb lead $\mathrm{Pb}$ (II) in water into a mesoporous silica coating that was deposited on a $\mathrm{SiO}_{2}$ covered $\mathrm{MZ}$ interferometer $(L=2.04 \mathrm{~mm})$.

The same sensor platform will also be used for sensing of other volatile organic compounds (VOCs) such as formaldehyde, other BTEX compounds (benzene, toluene, ethylbenzene and xylene), ozone, methylene chloride, trichloroethylen, and other VOCs that are indicative of poor indoor air quality. In addition to high sensitivity, the reversibility of the uptake process is essential for environmental monitoring applications and forms a departure from existing chemical sensors on micro-photonic platforms.

\section{REFERENCES}

[1] A. Densmore, D.-X. Xu, S. Janz, P. Waldron, T. Mischki, G. Lopinski, A. Delage, J. Lapointe, P. Cheben, B. Lamontagne, and J. H. Schmid, "Spiral-path high-sensitivity silicon photonic wire molecular sensor with temperature-independent response", Opt. Lett. 33, 596 (2008).

[2] A. Densmore, M. Vachon, D.-X. Xu, S. Janz, R. Ma, Y. H. Li, G. Lopinski, A. Delage, J. Lapointe, C. C. Luebbert, Q. Y. Liu, P. Cheben, and J. H. Schmid, "Silicon photonic wire biosensor array for multiplexed real-time and label-free molecular detection", Opt. Lett. 34, 3598 (2009).

[3] J. A Barnes, M. Dreher, K. Plett, R. S. Brown, C. M. Crudden, and H. P. Loock, "Chemical sensor based on a long-period fibre grating modified by a functionalized polydimethylsiloxane coating, Analyst 133, 1541 (2008).

[4] J. A. Barnes, R. S. Brown, A. H. Cheung, M. A. Dreher, G. Mackey, and H.-P. Loock, "Chemical sensing using a polymer coated long-period fiber grating interrogated by ring-down spectroscopy", Sensors Actuat. B-Chem., in print, (2010).

[5] J. Du, J. Cipot-Wechsler, J. M. Lobez, H.-P. Loock, and C. M. Crudden, "Periodic Mesoporous Organosilica Films: Key Components of Fibre Optic-Based Heavy Metal Sensors", Small, in press, (2010).

[6] R. Muzzalupo, G. A. Ranieri, G. Golemme, and E. Drioli, "SelfDiffusion Measurement of Organic Molecules in PDMS and Water in Sodium Alginate Membranes", J. Appl. Polymer Sci. 74, 1119 (1999).

[7] K. Wouters and R. Puers, "Accurate measurement of the steady-state swelling behavior of SU-8 negative photo resist", Proc. Eurosensors XXIII Conf., Procedia Chem., 1, 1, 60 (2009). 